\title{
PERFORMANCE OF A VERY HIGH VOLTAGE PHOTOEMISSION ELECTRON GUN FOR A HIGH BRIGHTNESS, HIGH AVERAGE CURRENT ERL INJECTOR*
}

\author{
B. M. Dunham, C. K. Sinclair, I. V. Bazarov, Y. Li, X. Liu, and K. W. Smolenski \\ Cornell University, Ithaca, NY 14853, U.S.A.
}

\begin{abstract}
We have constructed a very high voltage DC photoemission electron gun as the electron source of an injector for an Energy Recovery Linac (ERL) based synchrotron radiation light source. The gun is designed to deliver $100 \mathrm{~mA}$ average beam current in a $1300 \mathrm{MHz} \mathrm{CW}$ bunch train (77 pC/bunch), and to operate up to $750 \mathrm{kV}$ cathode potential. Negative electron affinity (NEA) photocathodes are used for their small thermal emittance and high quantum efficiency. A load-lock system allows introduction, cleaning, and activation of cathodes outside of the electron gun. Cathodes are cleaned by heating and exposure to atomic hydrogen, and activated with cesium and nitrogen trifluoride. Cathode electrodes of $316 \mathrm{LN}$ stainless and Ti4V6Al have been used with a beryllium anode. The internal surface of the ceramic insulator has a high resistivity fired coating, providing a charge drainage path. Non-evaporable getter (NEG) pumps provide a very high pumping speed for hydrogen. Operating experience with this gun will be presented.
\end{abstract}

\section{INTRODUCTION}

Cornell University is planning the construction of a synchrotron radiation light source based on an ERL. This light source will have a $100 \mathrm{~mA}$ average beam current in a $1300 \mathrm{MHz}$ CW train of $77 \mathrm{pC}$ bunches. With small beam emittances and short bunch durations from the injector, an ERL-based light source can deliver exceptional X-ray beam brightness, high coherent fluxes, and very short duration X-ray pulses. Cornell is presently funded to demonstrate an injector meeting the required average current, beam brightness, and bunch duration specifications for the proposed ERL light source.

We have chosen a very high voltage DC electron gun with an NEA photoemission cathode as the electron source of this injector. We judged the alternative technologies of normal conducting or superconducting RF guns to be either inconsistent with achieving high brightness, high average current $\mathrm{CW}$ beam (normal conducting), or based on relatively immature technology (superconducting). While no DC gun has approached the operational parameters we must reach, several very high voltage DC guns have been successfully operated. The Jefferson Lab FEL gun routinely operates at $350 \mathrm{kV}$, and has briefly delivered over $9 \mathrm{~mA}$ average current from a GaAs photocathode. Nagoya University has developed a $200 \mathrm{kV}$ gun for polarized electron beam delivery.

\footnotetext{
*Work supported by the National Science Foundation under contract
} PHY 0131508
A computational optimization has shown that a 500-750 $\mathrm{kV}$ DC photoemission gun can deliver our required bunch charge and duration with exceptionally small transverse and longitudinal emittances [1]. The transverse emittance of the beam from our full injector was shown to be dominated by the thermal emittance of the photocathode. This is a powerful argument for NEA photocathodes, which offer very small thermal emittances. Also, laser systems supporting the delivery of $100 \mathrm{~mA}$ average current with $1300 \mathrm{MHz}$ RF time structure from NEA photocathodes are technically feasible [2].

\section{TECHNICAL ISSUES}

Photocathode operational lifetime is the foremost challenge for a high average current photoemission electron gun. The best photoemission cathodes to date have delivered only a few hundred coulombs before they must be either cleaned and reactivated, or replaced. For a $100 \mathrm{~mA}$ average current, significantly better photocathode lifetimes must be achieved. Experiments at Jefferson Lab have shown ion back-bombardment to be the primary cause of NEA photocathode degradation [3]. The lowest possible pressure in the gun is thus key to obtaining good cathode operational lifetimes. Even at the lowest practical pressures of $10^{-9}$ to $10^{-10} \mathrm{~Pa}$, a high average current photocathode will need to be cleaned and reactivated or replaced regularly, leading to a design with a load-lock system for photocathode transfer between the gun and cleaning and activation systems.

Field emission (FE) from the cathode electrode structure can lead to charging of the ceramic insulator isolating the cathode voltage, and pressure rise from localized heating. In the worst case, these problems can lead to punch-through or flashover of the cathode insulator, breakdown of the cathode-anode gap, localized melting where FE electrons strike, and vacuum degradation. It is essential to minimize FE from the cathode electrode structure, and photon and ion feedback from the anode to the cathode.

For any linear photoemitter, the emission current is related to the incident optical power and quantum efficiency by

$$
i(m A)=\frac{\lambda(n m)}{124} \bullet P(W) \bullet Q E(\%)
$$

with $\lambda$ the illumination wavelength in $\mathrm{nm}$; $\mathrm{P}$ the optical power in Watts; and QE the cathode quantum efficiency at the illumination wavelength in percent. $100 \mathrm{~mA}$ average current from a photocathode illuminated at $525 \mathrm{~nm}$ 
requires a power-quantum efficiency product of $23.6 \mathrm{~W}$ $\%$. With green illumination, $28 \%$ of the incident power is absorbed in the GaAs. We measured the temperature rise of a GaAs wafer mounted on a thermally isolated molybdenum carrier ("puck") and illuminated by $20 \mathrm{~W}$ of green light to be $\sim 270 \mathrm{C}$. This temperature would quickly destroy the photocathode. The materials used for cathode electrode construction have very low thermal conductivity, so a cathode heat removal path separate from the electrode structure must be incorporated.

Finally, very high voltage power supplies require insulation, typically with pressurized $\mathrm{SF}_{6}$ gas. Care must be taken to assure that the very large forces involved do not misalign or deform the mounting of the cathode electrode structure, or cause failure of the vacuum seals or ceramic-to-metal braze joints.

\section{GUN DESIGN AND CONSTRUCTION}

Figure 1 shows a cutaway view of our gun design. The cathode electrode structure has ten separate pieces. Seven are exposed to electric field. The cathode wafer is mounted on a molybdenum puck, inserted into the Pierce cathode electrode by a translation mechanism, and held in place by an array of monel springs. The springs hold the puck against a tubular copper piece connected by a $5 \mathrm{~cm}$ diameter copper rod to the external environment, making a path for cathode heat removal. Electrode pieces exposed to high fields are polished with diamond paste and cleaned for ultrahigh vacuum. The electrode structure is assembled without touching any surface exposed to electric field. The structure is mounted to a spider bolted to the high potential end of the ceramic insulator, and aligned by shimming the individual legs of the spider. Ti4V6Al and 316LN stainless electrodes were fabricated.

The gun ceramic and electrode structure is physically large to reduce the peak electric fields. The highest field is on the rounded portion of the 25 degree Pierce cathode electrode, and is $13.6 \mathrm{MV} / \mathrm{m}$ at $500 \mathrm{kV}$. Field strengths outside this small region are below $11 \mathrm{MV} / \mathrm{m}$ at $500 \mathrm{kV}$. The capacitance of the complete gun is less than $\sim 100 \mathrm{pF}$, and the power supply adds an additional $\sim 24 \mathrm{pF}$, leading to a total stored energy of 15.5 Joules at $500 \mathrm{kV}$.

The beryllium anode is mounted on a copper plate. Beryllium offers high thermal conductivity, low radiative yield, and a large range for cathode potential electrons. These desirable properties greatly reduce photon and ion feedback from the anode to the cathode.

The large ceramic insulator, fabricated by CPI, has a proprietary resistive coating on its inner surface. This coating gives a fairly uniform sheet resistance, with a value adjustable by multiple coatings and firings. At high temperature the coating diffuses into the ceramic, providing electrical conductivity over a depth comparable to the range of cathode potential electrons. We have chosen an end-to-end resistance of about $50 \mathrm{GOhm}$ at 500 $\mathrm{kV}$, leading to a $10 \mu \mathrm{A}$ current flow over the inner surface of the ceramic. This current is adequate to sink moderate FE currents from the electrode structure. Two ceramics

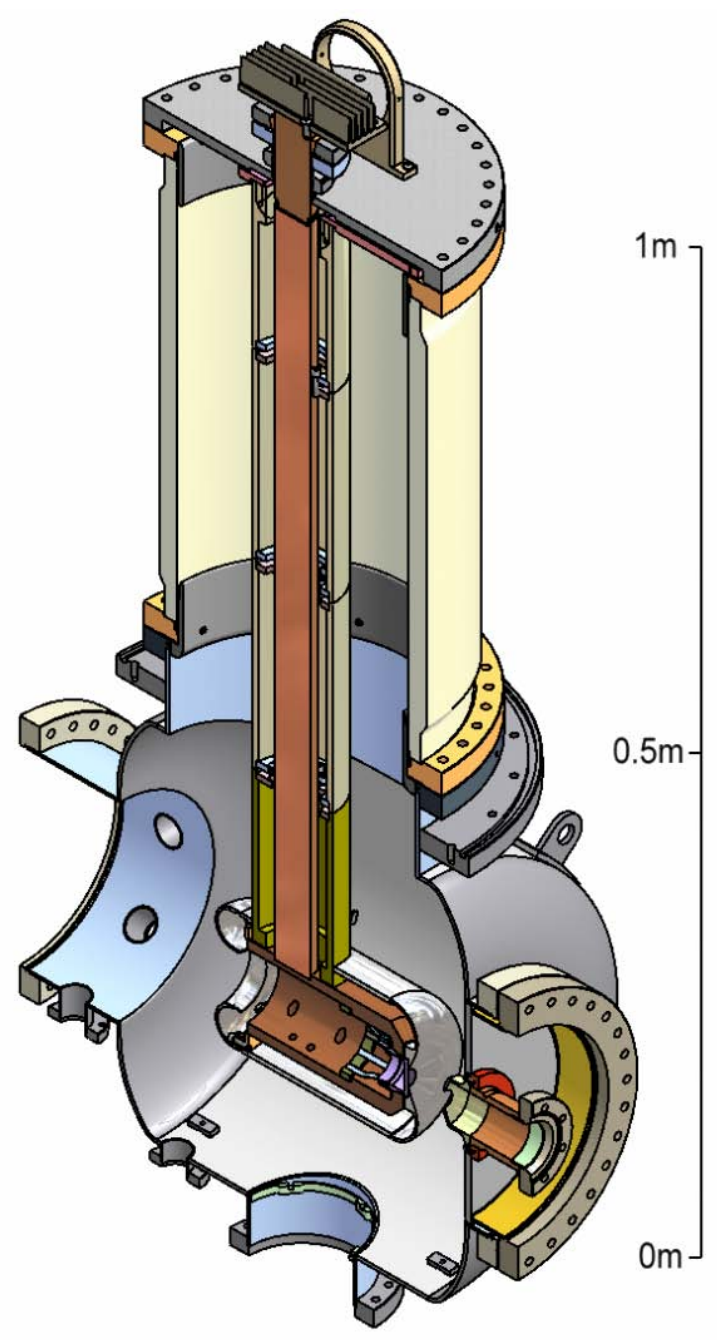

Figure 1. A cutaway view of the electron gun

were fabricated. The first, used during the gun testing reported here, had a much higher end-to-end resistance.

The gun chamber is $316 \mathrm{~L}$ stainless, with $316 \mathrm{LN}$ stainless flanges, allowing it be vacuum fired to $950 \mathrm{C}$. The gun is assembled on a thermally insulating table, with thermally insulating walls added for a bakeout enclosure. An RF shielded all metal gate valve is immediately downstream of the anode, and an unshielded all metal valve isolates the gun chamber from the load-lock system. Roughing is done with a dry pumping system, through a line with all metal valves. A $40 \mathrm{l} / \mathrm{s}$ ion pump external to the baked volume serves as the primary pump during bakeout. The gun chamber is pumped by a $400 \mathrm{l} / \mathrm{s}$ DI ion pump and a total of twenty commercial NEG pump modules (not shown in Fig. 1). Fully activated, the NEGs provide a total hydrogen pumping speed of $\sim 22,000 \mathrm{l} / \mathrm{s}$. Vacuum diagnostics are a quadrupole RGA with electron multiplier and an extractor gauge. The RGA filament is distant from all chamber walls, but this was not practical with the extractor gauge. The gun chamber and the $316 \mathrm{LN}$ electrode set were fired in air for 100 hours at 400 $\mathrm{C}$ to reduce the outgassing rate. The gun is baked to only 
$165 \mathrm{C}$. These procedures are reported to produce a very low hydrogen outgassing rate [4].

The $750 \mathrm{kV}, 100 \mathrm{~mA}$ cathode power supply employs proprietary insulating core transformer technology [5]. Its high voltage section is insulated by $\mathrm{SF}_{6}$ gas at 5 bar. The gun and the high voltage section are contained within a code-certified stainless pressure vessel, and connected by an aluminum tube during beam operation, or a $75 \mathrm{MOhm}$ conditioning resistor for high voltage processing. The $\mathrm{SF}_{6}$ pressure vessel is supported from the ceiling on four springs. The primary of the insulating core transformer is driven by an external $800 \mathrm{~V}$ p-p high frequency, pulse width modulated power supply, which receives power from a $480 \mathrm{VAC}, 3$ phase power conditioner.

GaAs or GaAsP cathode wafers are indium soldered to molybdenum pucks. An annular region on each wafer is anodized to eliminate photoemission from near the Pierce electrode. Pucks are introduced into a small load-lock chamber, which is evacuated and baked prior to moving the pucks into the rest of the system through an all metal valve. Cathodes are cleaned by heating and exposure to atomic hydrogen, and transferred into a third chamber for activation with $\mathrm{Cs}$ and $\mathrm{NF}_{3}$. During cleaning, the activation chamber is isolated by a conductance restriction valve. One cathode can be stored in the activation chamber while a second is in the gun. Two orthogonal $0.91 \mathrm{~m}$ long bellows translation mechanisms move the pucks between the three load-lock chambers, and between the activation/storage chamber and the gun cathode structure. The load-lock system is constructed on a thermally insulating table, with thermally insulating walls added for bakeout. Heat for baking the gun and the loadlock system is from high power hot air guns. The loadlock system is mechanically isolated from the gun by a bellows. Alignment of the load-lock system to the gun axis is done by optical survey methods.

\section{CURRENT STATUS}

The gun and load-lock system have been operated over the past nine months, with a number of interruptions to understand and resolve various problems. Both electrode sets have been used. The titanium set performed poorly, traced in part to contamination by metallic flakes believed to arise from the silver-plated bolts used in the gun. The stainless steel electrode set has shown significant field emission above $300 \mathrm{kV}$, and has processed only slowly above this voltage. The present ceramic has a very high end-to-end resistance, and had a punch-through failure at $330 \mathrm{kV}$, so beam operations have been restricted to 275 $\mathrm{kV}$, where $\mathrm{FE}$ is undetectably small. Beam operations have concentrated on quantitative verification of the performance of the beam diagnostics, and on cathode thermal emittance measurements [6]. An attempt to increase the average beam current was limited above 5 $\mathrm{mA}$ by pressure rise from the beam dump.

After activation of the NEG pumps, the vacuum in the gun is excellent. The $400 \mathrm{l} / \mathrm{s}$ ion pump current settled at $1.25 \mathrm{nA}$, implying a pressure in the low $10^{-10} \mathrm{~Pa}$ range, while the extractor gauge settled at about $6 \times 10^{-10} \mathrm{~Pa}$. Correcting this for hydrogen would imply a pressure of about $1.5 \times 10^{-9} \mathrm{~Pa}$. The residual gas is completely dominated by hydrogen. We believe the extractor gauge reads high due to its filament being close to nearby vacuum chamber walls, and that the static pressure in the gun is in the mid $10^{-10} \mathrm{~Pa}$ range.

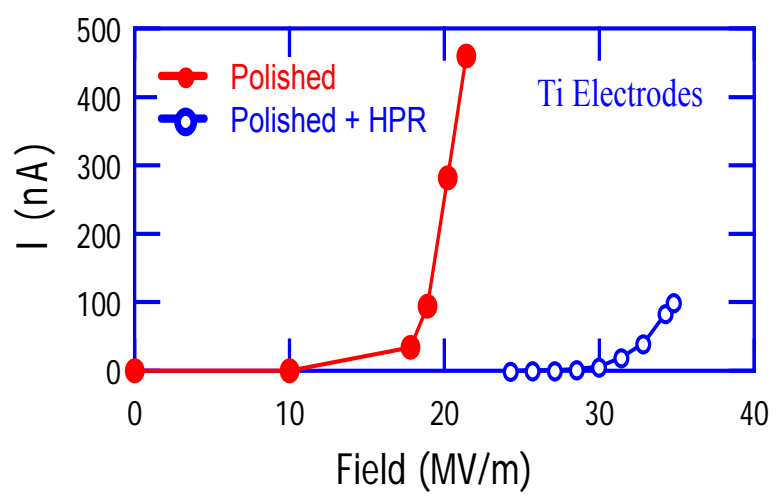

Figure 2. FE from a polished titanium electrode before and after HPR treatment

We have continued to study reduction of FE from large area electrodes in a separate ultrahigh vacuum chamber. Recently, we used high pressure water rinsing (HPR) to remove particulate contamination from test electrodes, with considerable success. HPR greatly reduces FE in superconducting RF cavities, and is now in wide use for this purpose. Figure 2 shows the change in FE obtained by HPR of a titanium alloy test electrode. Similar results have been obtained with several other samples. The electrodes used for these tests had a uniform field area of $>116 \mathrm{~cm}^{2}$, and a gap of $3.5 \mathrm{~mm}$.

A new ceramic insulator with much lower resistivity has been fabricated, and the gun will be rebuilt with it during the summer. With this and HPR treatment of the electrodes, we believe we will reach the higher gun voltages required to meet the specifications of the ERL electron source. We have added conductance restrictions and NEG coatings on the beam pipes to reduce the gas reaching the gun from dump outgassing, which should allow operation at higher average current.

\section{REFERENCES}

[1] I.V. Bazarov and C.K. Sinclair, PRST-AB 8 (2005) 034202

[2] D.G. Ouzounov et al., "The laser system for the ERL electron source at Cornell University", these proceedings.

[3] C.K. Sinclair et al., PRST-AB 10 (2007) 023501

[4] M. Bernardini et al., JVST A 16, 188 (1998)

[5] Manufactured by Kaiser Systems, Inc., Beverly MA

[6] I.V. Bazarov et al., "Thermal emittance measurements from negative electron affinity photocathodes", these proceedings. 